\title{
Contextual Radiology Reporting: A New Approach to Neuroradiology Structured Templates
}

\author{
(D) M.D. Mamlouk, DP.C. Chang, and (DR.R. Saket
}

it

\begin{abstract}
SUMMARY: Structured reporting has many advantages over conventional narrative reporting and has been advocated for standard usage by radiologic societies and literature. Traditional structured reports though are often not tailored to the appropriate clinical situation, are generic, and can be overly constraining. Contextual reporting is an alternative method of structured reporting that is specifically related to the disease or examination indication. Herein, we create a library of 50 contextual structured reports for neuroradiologists and emphasize their clinical value over noncontextual structured reporting. These templates are located in the On-line Appendix, and a downloadable PowerScribe 360 file may be accessed at https://drive.google.com/open?id=1AlPUmfAXPzjkMFcHf7vGKF4Q-vldpflT.
\end{abstract}

ABBREVIATIONS: MAGNIMS = Magnetic Resonance Imaging in Multiple Sclerosis; PHACE = posterior fossa malformations, hemangiomas, arterial anomalies, cardiac defects, and eye abnormalities

$\mathbf{R}$ ecent literature and several radiology organizations advocate the use of structured templates as a new standard for radiology reporting. ${ }^{1-5}$ Structured reporting has many advantages over traditional free-text, narrative reporting, including improved consistency, increased clarity, decreased grammar and speech recognition errors, increased ability to mine data for research, and greater financial rewards. ${ }^{6-10}$ On the other hand, advantages of narrative or prose reporting include flexibility, personalization, and familiarity for radiologists. While structured reporting is not perfect, referring providers have expressed greater satisfaction with the implementation of structured reporting. ${ }^{8}$ Despite this impetus, there are few data that objectively demonstrate the added clinical value over narrative reporting. ${ }^{11}$

Received January 19, 2018; accepted after revision April 20.

From the Department of Radiology (M.D.M., R.R.S.), The Permanente Medical Group, Kaiser Permanente Medical Center Santa Clara, Santa Clara, California; Department of Radiology and Biomedical Imaging (M.D.M., R.R.S.), University of California, San Francisco, San Francisco, California; and Department of Radiology (P.C.C.), The Permanente Medical Group, Kaiser Permanente Medical Center South San Francisco, South San Francisco, California.

Paper previously presented as an education exhibit at: Annual Meeting of the Radiological Society of North America, November 26-December 1, 2017; Chicago, Illinois. It received the Magna Cum Laude award.

Please address correspondence to Mark D. Mamlouk, MD, Department of Radiology, The Permanente Medical Group, Kaiser Permanente Medical Center Santa Clara, 700 Lawrence Expressway, Santa Clara, CA 95051; e-mail: mark.d.mamlouk@kp.org, mark.mamlouk@ucsf.edu; @MarkMamloukMD

- Indicates open access to non-subscribers at www.ajnr.org

三 Indicates article with supplemental on-line appendix.

Indicates article with supplemental on-line photos.

http://dx.doi.org/10.3174/ajnr.A5697
Not all structured reporting is created equally. Many of the published structured templates are technique- or examinationbased. ${ }^{3}$ For example, in CT abdomen and pelvis imaging, structured reports are organized by a litany of organs. ${ }^{3}$ Similarly within neuroimaging, a sample published MR imaging brain template contains 16 anatomic items within the findings section, and nearly all the fields are filled in with "normal." 12 While these reports may be versatile, they do not guide the radiologist during image interpretation but simply provide fields to report in a "structured" fashion. In addition, some radiologists are reluctant to use structured reporting because they think that the reports are too generic or simplistic and often not appropriately tailored to the clinical scenario. ${ }^{13}$

\section{Contextual Structured Reporting}

Contextual reporting is an alternative method of structured reporting that is specifically related to the disease or examination indication. These disease-specific reports provide content focused on the clinical diagnosis or symptom, discuss appropriate differential diagnoses, and highlight pertinent positives and negatives. Contextual reports are flexible; pick lists can be created within the templates to permit different options for the radiologist. Depending on the reporting software, tiered concepts can also be managed to trigger other macros that can be inserted on the basis of specific keywords or selection of pick lists. Contextual reporting also does not preclude some free-text dictation within the appropriate contextual fields. No template can perfectly fit every clinical scenario; thus, contextual templates should not be overly restrictive. This feature is another negative that some radiologists have found with traditional structured reporting. ${ }^{14}$ 
Contextual structured reporting has only been applied to a handful of diseases, including pancreatic cancer, pulmonary embolism, pediatric Crohn disease, prostate cancer, rectal cancer, infertility, and interstitial lung disease. ${ }^{11,15-20}$ To the authors' knowledge, contextual templates for multiple sclerosis, thyroid imaging, and head trauma are the only contextual structured reports developed within neuroimaging. ${ }^{21-23}$ The purpose of this article was to create a library of 50 contextual structured reports for neuroradiologists and emphasize their clinical value over noncontextual structured reporting through the use of selective imaging examples.

\section{Template Creation Process}

The contextual templates were created in a systematic process that spanned $>1$ year, which is similar to processes in other institutions. ${ }^{24,25}$ Our institution (Kaiser Permanente, Santa Clara, California) is part of a 21-hospital network comprising 300 radiologists, 50 of whom are neuroradiologists. Led by a committee chairperson, a committee search was initiated to identify radiologists interested and knowledgeable about structured reporting and informatics. The subspecialty regional leaders of neuroradiology within brain, neurovascular, head/neck, pediatric neuroradiology, and spine imaging were recruited. After the committee was created, common examination indications and diseases within neuroradiology were culled from examination data, and templates were drafted. For each template created, the committee members provided revisions and ensured that the templates contained content contextual to the disease process or examination indication. Following committee voting and approval, each of the templates was sent to a subspecialty clinician who was an expert in the specific topic to provide additional suggestions. Before template publication within our radiology dictation software (Radiator; Napa, California), the informatics leader of the committee coded the template, ensuring the templates were practical to use and creating pick list options when appropriate.

The templates were mapped to the procedural codes of our institution within the radiology information system, which are closely modeled after the current procedural terminology codes, with the exception of more granularity to permit more complex associations between the procedures and structured templates. This mapping permits the template to automatically load in the reporting software when the examination is opened for interpretation. The templates were then made available to all radiologists to use in clinical practice, and a hyperlink was inserted within the dictation software for users to provide suggestions for change, which were reviewed and revised when appropriate. To orient the radiologists with the contextual templates at the onset of deployment, the template committee gave a lecture on the types of templates developed, the advantages of contextual reporting, and reference to available imaging guides for certain templates.

For the purposes of this publication and to make the contextual templates easily accessible to other radiologists without our proprietary reporting software, the templates were also created in Nuance Powerscribe360 (Burlington, Massachusetts). A downloadable Powerscribe 360 file can be accessed here (https://drive.
google.com/open?id=1AlPUmfAXPzjkMFcHf7vGKF4QvIdpflT) and is compatible with versions 3.5 and 4.0 .

\section{Contextual Structured Reporting Templates}

The advantages of contextual templates are 3-fold: They are individually tailored to the diagnosis, they ensure that all pertinent points are addressed in a checklist fashion, and they educate trainees by providing a systematic approach for clinical interpretation.

\section{Contextual Templates Individually Tailored to the Diagnosis}

Multiple Sclerosis Screening. Our institution abides by the Magnetic Resonance Imaging in Multiple Sclerosis (MAGNIMS) consensus guidelines for diagnostic and follow-up imaging. ${ }^{26,27} \mathrm{MR}$ imaging criteria to establish disease dissemination in space are characterized as having at least 2 of the 5 findings: $\geq 3$ periventricular lesions, $\geq 1$ infratentorial lesion, $\geq 1$ spinal cord lesion, $\geq 1$ optic nerve lesion, and $\geq 1$ cortical/juxtacortical lesion. These specific criteria render them suitable for a contextual template (Fig 1), one that is focused solely on multiple sclerosis and is supported by evidencebased medicine. This template is used in screening examinations with a unique imaging screening protocol to determine whether the patient has multiple sclerosis. At the end of the template, the MAGNIMS criteria are listed for a convenient reminder to the ordering provider. This contextual multiple sclerosis template addresses items similar to those in the previously reported multiple sclerosis templates, ${ }^{21,22}$ though the actual templates in these articles are not published for definite comparison.

Multiple Sclerosis Follow-Up. Evaluating disease progression or stability in the follow-up of multiple sclerosis can sometimes be cumbersome to the radiologist, especially when the disease burden is high. Because of this occasional difficulty, reporting can also be vexing. On the other hand, contextual reporting for multiple sclerosis has been anecdotally the easiest and most rapid template to fill out because it mainly focuses on the presence of new or active demyelinating lesions (Fig 2). Many of the fields can be quickly tabbed through, and a final report can often be crafted in a few minutes or less after reviewing the images.

Head and Neck Cancer. Reporting examinations for head and neck cancer warrant a thorough discussion of the primary tumor and nodal metastases. Contextual templates can assist with reporting by directing the radiologist to the pertinent findings relevant to staging by the American Joint Committee on Cancer. The template, which can be used for both MR imaging and CT, is divided into 2 sections: tumor and nodal characteristics (Fig 3), which provide the referring provider a clear and focused assessment. Also, by reporting in a systematic fashion, this template enables the radiologist to synthesize the findings and include the clinical staging in the impression section of the report. While some radiologists and institutions may not prefer to include the clinical staging within their radiology reports, it is still paramount to report all the pertinent positives and negatives so that the clinical stage can be established by the referring provider or within the hospital tumor board. As a final note, initially, contextual templates were created for each of the neck cancer sites; however, given the various presentations of each tumor and the several structures potentially 
FINDINGS:

BRAIN PARENCHYMA:

T2 hyperintense white matter lesions:

*Periventricular: [No|1-2|3 or more] lesions contacting the ependymal surface

*Juxtacortical/Cortical: [None|Present]

*Infratentorial: [None|Present involving the [brainstem|cerebellum|brainstem and cerebellum]]

*Optic Nerve: [None|Present]

*Cervicomedullary Junction: [None|Present]

Enhancing Lesions: [ $<\#$ of enhancing lesions and locations $>$ None]

Reduced Diffusion: [None|Present (describe)]

Overall Disease Burden: [None $\mid<10$ lesions $\mid 10-20$ lesions $\mid>20$ lesions]

Parenchymal Atrophy: [None|Mild|Moderate|Severe]

Callosal Atrophy: [None|Mild|Moderate|Severe]

OTHER FINDINGS: [None]

IMPRESSION:

[Normal MRI brain.|Multiple white matter lesions that are [typical|atypical|not consistent] with demyelinating disease.]

2016 MAGNIMS MRI criteria to establish disease dissemination in space in multiple sclerosis (Lancet Neurol. 2016 Mar;15(3):292-303)

Involvement of at least two of five areas of the CNS as follows:

* Three or more periventricular lesions

* One or more infratentorial lesion

* One or more spinal cord lesion

* One or more optic nerve lesion

* One or more cortical or juxtacortical lesion

FIG 1. Contextual template for multiple sclerosis screening. The template is individually tailored to the diagnosis of multiple sclerosis and is evidence-based, abiding by MAGNIMS criteria.

\section{FINDINGS:}

BRAIN PARENCHYMA:

New T2 hyperintense Lesions: [<new lesion number and location $>$ None]

Enhancing Lesions: [ $<\#$ of enhancing lesions and locations $>$ None]

Reduced Diffusion: [None|Present (describe)]

Overall Disease Burden: [ $<10$ lesions $\mid 10-20$ lesions $\mid>20$ lesions]. [No change in multiple [periventricular|periventricular and juxtacortical|periventricular and infratentorial|periventricular, juxtacortical and infratentorial] white matter lesions compatible with known demyelinating disease].

T1 Hypointensities (Black Holes): [Absent $|<5|>5$ ] and [unchanged|increased] compared to prior exam].

Parenchymal Atrophy: [None|Mild|Moderate|Severe]

Callosal Atrophy: [None|Mild|Moderate|Severe]

OTHER FINDINGS: [<other>None]

\section{IMPRESSION:}

Demyelinating disease in the [supratentorial|supratentorial and infratentorial] brain, [without|with] active disease and [no change|change] compared to $[<$ PriorExamDate $>$.

FIG 2. Contextual template for multiple sclerosis follow-up. Because the template focuses on items germane to the clinical indication, reporting can be accomplished rapidly, permitting quick tabbing through the fields. involved in each case, the templates were found to be constraining and time-consuming to report. Therefore, a single neck cancer template was created to provide report uniformity but also radiologist reporting flexibility.

Fetal Brain MR Imaging. Fetal neuroimaging naturally poses unique items for the radiologist to inspect and report that are different from those in postnatal imaging. Contextual structured reports for fetal MR brain imaging consist of 3 main areas: fetal positioning, biometry, and brain parenchyma (On-line Fig 1). Many of the items can be quickly selected through pick list options, which is an inherent advantage of structured reporting. In the event of a major fetal anomaly, these findings can be added to the corresponding field.

\section{Contextual Templates Ensuring All Pertinent Points Addressed in a Checklist Fashion}

Preoperative Sinus CT. Detecting sinonasal variants is an important assessment on preoperative sinus CT before endoscopic surgery. Despite published literature on sinonasal variants, radiology reporting of these variants is highly variable. Consequently, some otolaryngologists are dissatisfied with radiology reporting in this setting and even caution other otolaryngologists not to rely on the radiology report to manage their patients. ${ }^{28}$ This reporting variability is likely, in part, attributed to the difficulty in remembering all the sinonasal variants. A contextual preoperative sinus template helps with this dilemma by providing a checklist approach to remind the user of all the pertinent points (Fig 4). Our group has also added a hyperlink in our dictation software with a concise imaging guide of all the described sinonasal variants for easy access while reporting.

Preoperative Pituitary MR Imaging. A presurgical checklist is also important in pituitary surgery because certain anatomic variants can greatly impact the surgical approach. A discussion of the pituitary lesion is common throughout radiology reporting, though anecdotal experience from our surgeon colleagues reveals that radiologists do not often re- 

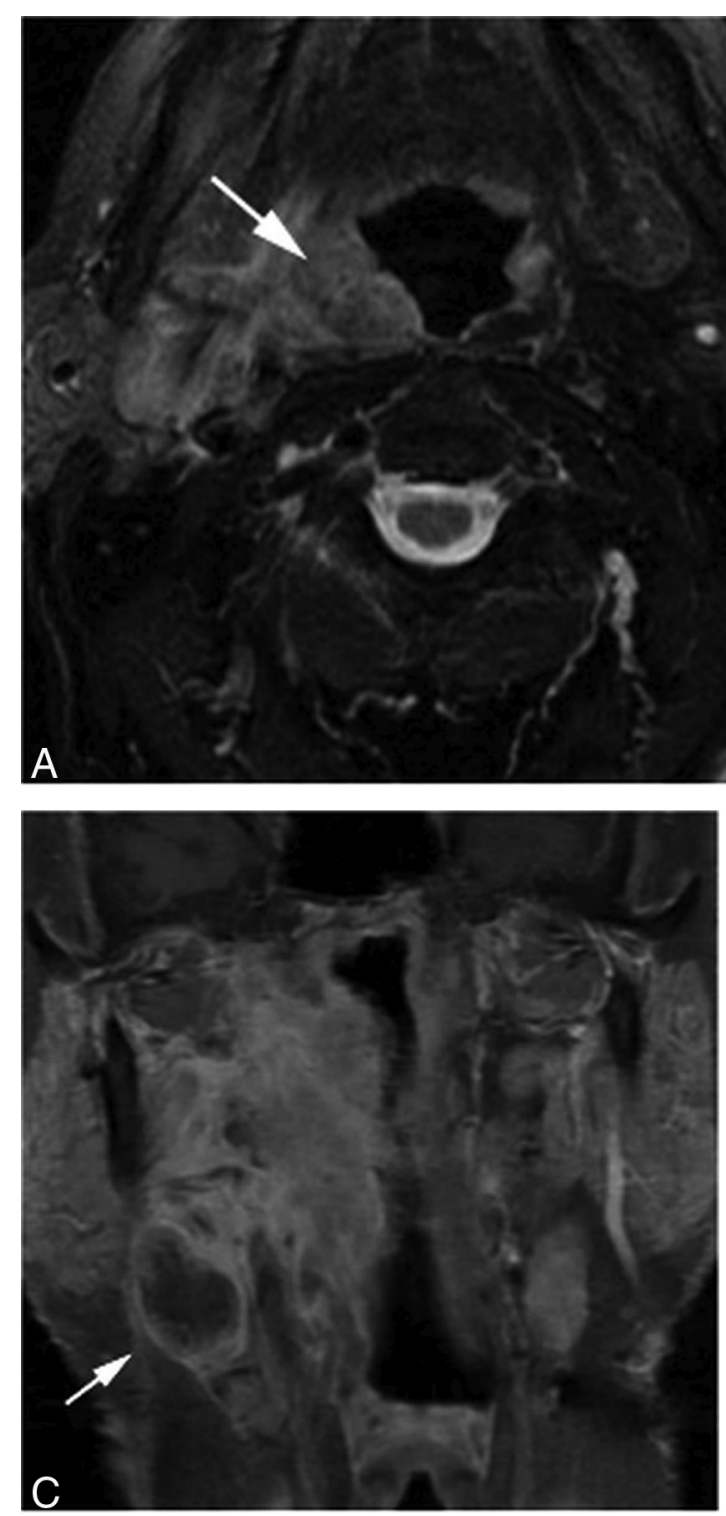

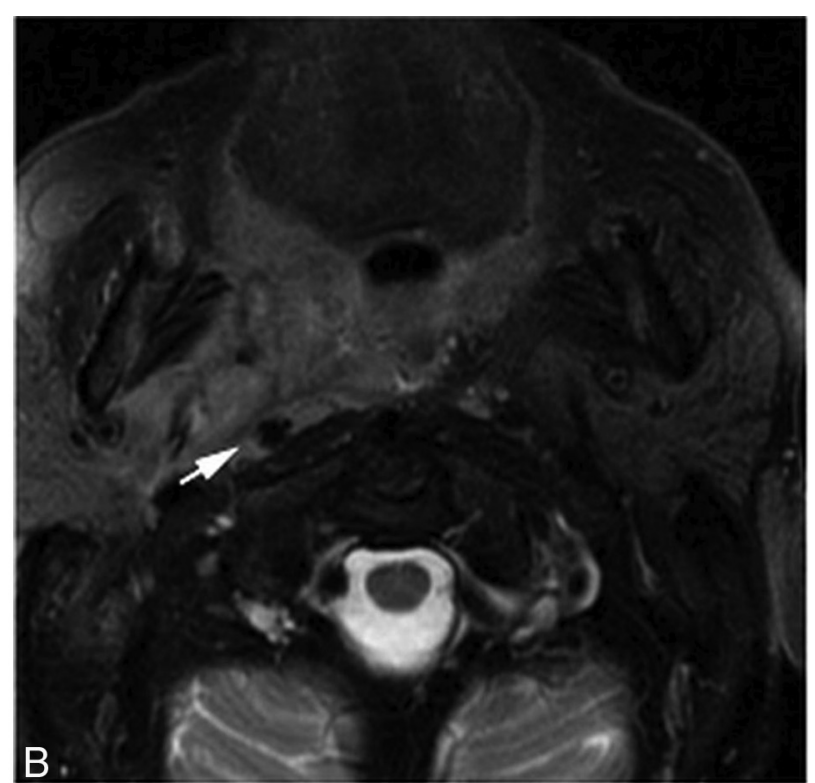

CLINICAL HISTORY: Right nodal biopsy = squamous cell carcinoma, HPV p16+

FINDINGS:

TUMOR:

Location: Right palatine tonsil

Size: $4.2 \times 3.2 \times 4.1 \mathrm{~cm}$

Description: Heterogeneous $\mathrm{T} 2$ enhancing lesion in the right palatine tonsil that extends to the contralateral tonsil. Epiglottis is spared.

Bony Invasion: Absent

Vascular Encasement: Present, encases R ICA

Perineural Spread: Absent

SUSPICIOUS LYMPH NODES: Present

Side/Level(s): Right level II

Largest Node: $3.1 \mathrm{~cm}$ right level II

Extracapscular Extension: Absent

Other Findings: Necrotic

\section{IMPRESSION:}

1. Right palatine tumor that measures $4.2 \mathrm{~cm}$ and encases $\mathrm{R}$ ICA.

2. Right level II $3.1 \mathrm{~cm}$ necrotic lymph node.

3. AJCC $8^{\text {th }}$ ed Staging: T4N1M0.

FIG 3. Value of a contextual template for neck cancer. $A$ and $B$, Axial fat-suppressed T2-weighted images show a right palatine tumor (long arrow) encasing the right internal carotid artery (short arrow), while $C$ shows a right level II node (arrow) on coronal contrast-enhanced fat-suppressed T1-weighted imaging. D, A contextual neck cancer template with all the fields concisely filled out and tailored to clinical staging.

port anatomic variants in this setting. Similar to the presurgical sinus CT template, a contextual pituitary template can establish a checklist reminder for the interpreting radiologist (On-line Fig 2). Anatomic variants discussed within the template include the following: aberrant vasculature such as persistent trigeminal artery, a narrowed intercarotid distance, sphenoid sinus pneumatization, and the intersphenoid septum. In the event that these items are not well evaluated on the interpreting MR imaging, review of prior imaging can usually provide confirmation, if available. Depending on the surgeon's preference, the extent of the pituitary lesion, and the patient anatomy, a neuronavigation CT can be performed afterward. Nonetheless, these items within the contextual MR imaging template can provide an initial framework for the surgical approach. For this template, our institution also created a short summary guide of the anatomic variants accessible within the dictation software to aid the interpreting radiologist.

Trigeminal Neuralgia. The imaging work-up of trigeminal neu- ralgia necessitates careful inspection for pathologies extending from the brain stem to the mandible. A contextual template facilitates this detailed evaluation in a succinct manner. The radiologist will remember where to identify potential causes, and the referring provider will be satisfied that the main diagnostic causes were assessed. Conversely, traditional free text or noncontextual structured templates could omit commentary on important elements and could also address unnecessary items, thereby cluttering the report. Figure 5 highlights this difference using a noncontextual structured template ${ }^{12}$ versus a contextual template for trigeminal neuralgia.

\section{Contextual Templates Educate Trainees by Providing a Systematic Approach for Clinical Interpretation}

Developmental Delay, Dementia, and Pulsatile Tinnitus. Certain entities, such as developmental delay, dementia, and pulsatile tinnitus, have several common etiologies that the radiologist must 

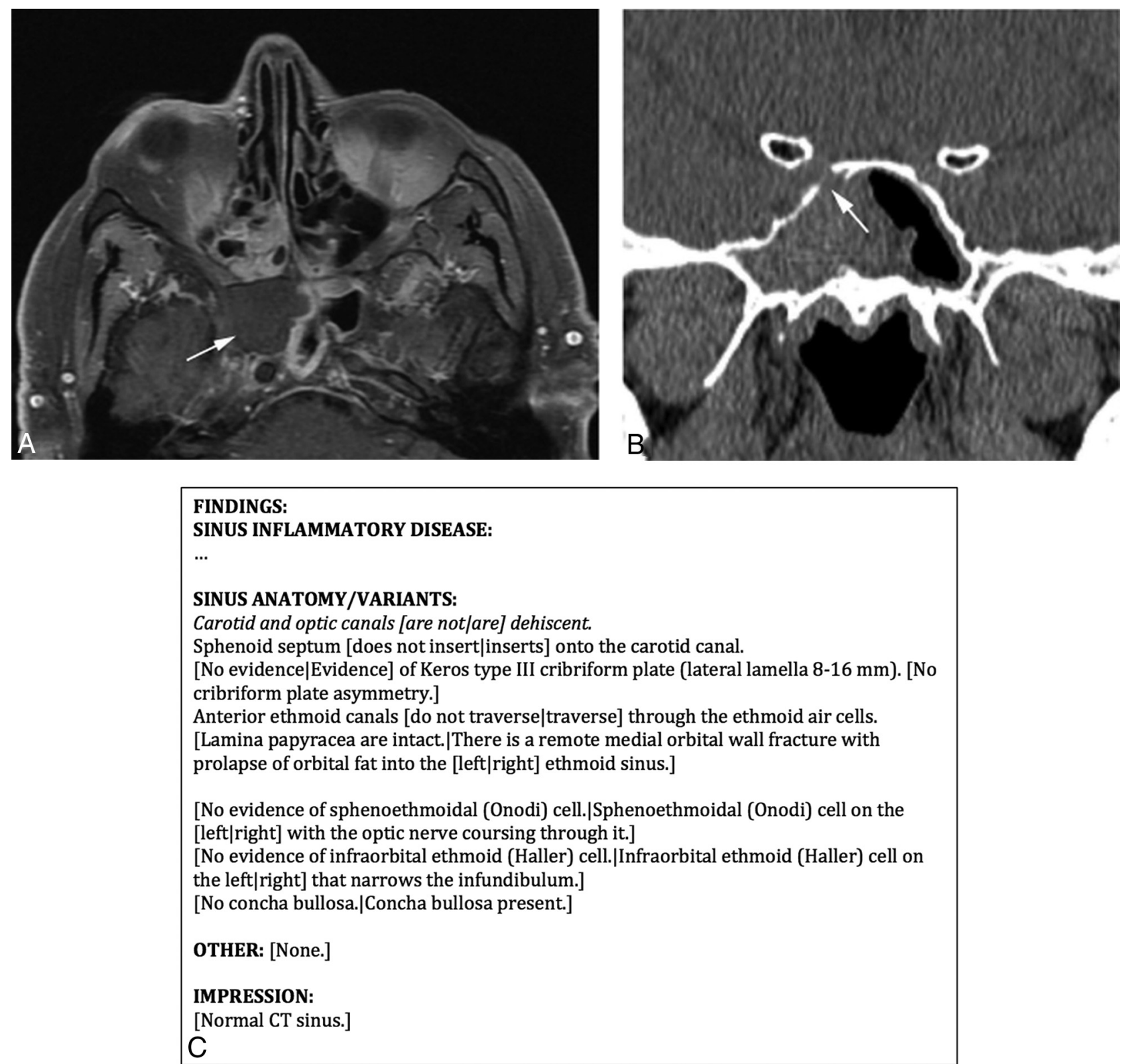

[No evidence of sphenoethmoidal (Onodi) cell.|Sphenoethmoidal (Onodi) cell on the [left|right] with the optic nerve coursing through it.]

[No evidence of infraorbital ethmoid (Haller) cell.|Infraorbital ethmoid (Haller) cell on the left|right] that narrows the infundibulum.]

[No concha bullosa.|Concha bullosa present.]

OTHER: [None.]

IMPRESSION:

[Normal CT sinus.]

C

FIG 4. Value of a contextual template for preoperative sinus CT. A, Axial contrast-enhanced fat-suppressed Th-weighted image shows diffuse paranasal sinus opacification, with nonenhancement in the right sphenoid sinus (arrow), compatible with invasive sinusitis. Preoperative sinus CT imaging was performed $(B)$ and shows a dehiscent right carotid canal (arrow) that could have been overlooked without a contextual template $(C)$. Remembering all the sinus variants can be difficult, but a contextual template can aid in this dilemma by providing a checklist approach. To improve education and usage of this template, a hyperlink can be added to the dictation software with imaging examples showing these sinonasal variants.

systematically inspect on imaging. Without a reference or guide, it is foreseeable that trainees may not be able to identify the important findings for these entities and synthesize them into a cohesive process. Contextual templates satisfy this need by serving as an adjunct guide to the attending radiologist. In fact, the instructing radiologist can teach trainees a systematic diagnostic approach to such cases with the template viewed in tandem with the imaging (Fig 6 and On-line Figs 3 and 4). The contextual templates will help reinforce knowledge that trainees need for their careers and board examinations.

This method will also educate trainees on reporting. It has been reported that radiology residents receive no more than 1 hour of didactic instruction in radiology reporting per year. ${ }^{29}$ Contextual templates can help with this deficient teaching by pro- viding trainees with a solid foundation on effective radiology reporting.

Last, contextual templates that emphasize a systematic approach to interpretation can help mitigate the cognitive bias of "satisfaction of search." ${ }^{30}$ This error occurs when a radiologist identifies a finding and overlooks additional findings due to complacency. Contextual reporting will remind the user to look at all described entities regardless of whether 1 finding is already identified.

\section{Contextual Templates for "Bread and Butter" Neuroradiology}

Many examinations in neuroradiology are performed for routine conditions such as stroke CT, low back pain, and cervical radiculopathy. We created templates for these 3 entities as well (On-line Figs 5-7). 

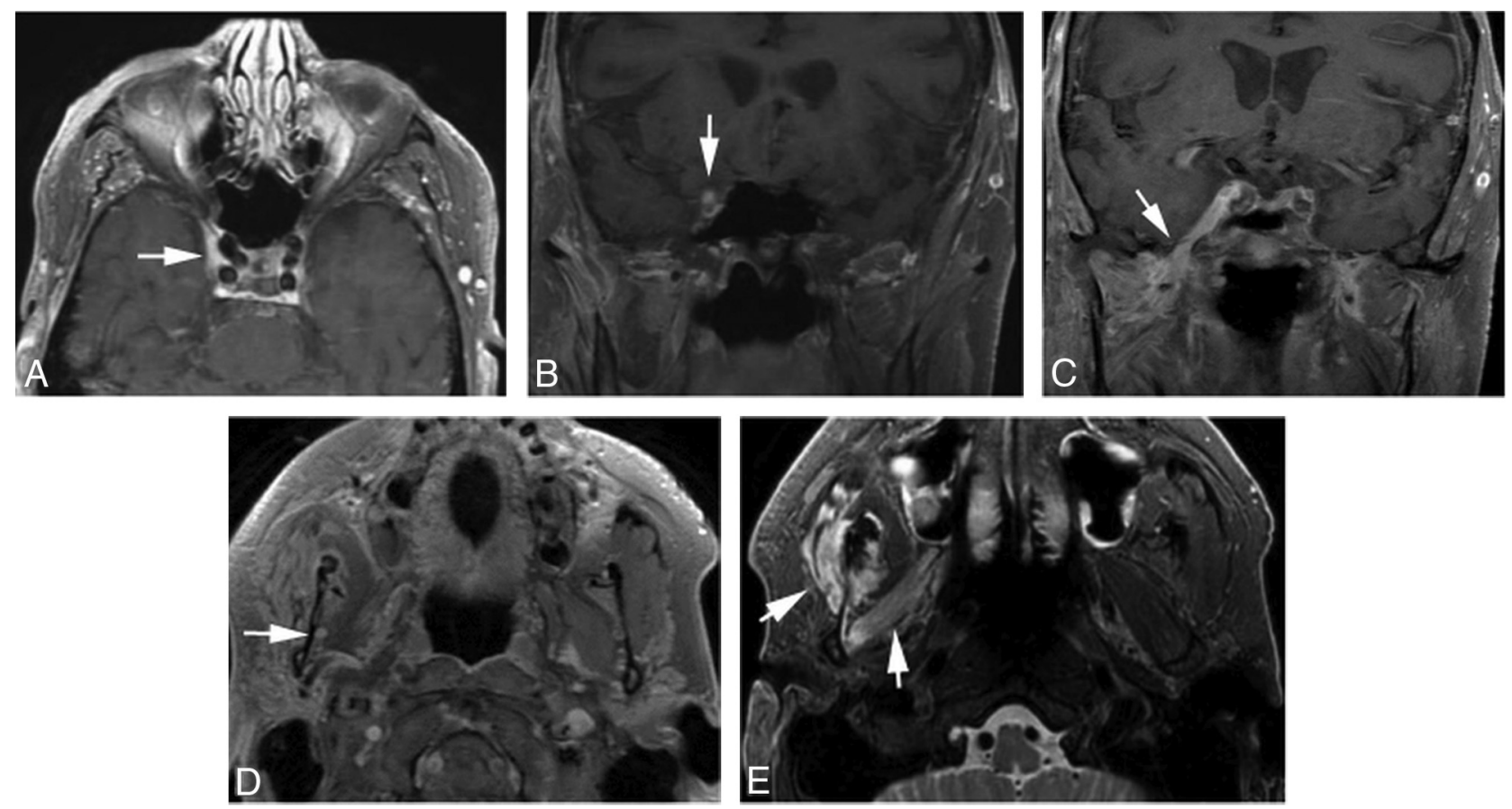

\section{FINDINGS:}

Extra-axial spaces: Normal in size and

morphology for the patient's age.

Intracranial hemorrhage: None.

Ventricular system: Normal in size and

morphology for the patient's age.

Basal cisterns: Normal.

Cerebral parenchyma: Normal.

Midline shift: None.

Cerebellum: Normal.

Brainstem: Normal.

Calvarium: Normal.

Vascular system: Normal flow voids, without

atheroma, occlusion, or dissection.

Paranasal sinuses and mastoid air cells: Clear.

Visualized orbits: Normal.

Visualized upper cervical spine: Normal.

Sella: Normal.

Skull base: There is abnormal enhancement

within the right cavernous sinus, foramen

rotundum, and foramen ovale.

Marrow: Normal.

\section{IMPRESSION:}

Abnormal enhancement within the right cavernous sinus, foramen rotundum, and foramen ovale, compatible with perineural spread of tumor from skin cancer.

$\mathrm{F}$

FINDINGS
TRIGEMINAL NERVE
Vascular Loop: Absent.
Posterior Fossa Mass: Absent.
Pons: No mass, acute infarct, or demyelinating lesion.
Perineural Enhancement: Abnormal enhancement within
the right foramen rotundum and foramen ovale.
Cavernous Sinus: Abnormal enhancement on the right.
Mandible: Enhancement and enlargement of the right
inferior alveolar nerve.
Muscles of Mastication: Right-sided denervation atrophy.
REMAINING BRAIN PARENCHYMA: No acute infarct or
hemorrhage. No mass effect or herniation.
VENTRICLES/EXTRA-AXIAL SPACES: No hydrocephalus or
extra-axial fluid collections.
IMPRESSION:
1. Abnormal enhancement within the right cavernous sinus,
foramen rotundum, foramen ovale, and inferior alveolar
nerve, compatible with perineural spread of tumor from
skin cancer.
2. Denervation atrophy of the right muscles of mastication.
G

FIG 5. Value of a contextual structured template for trigeminal neuralgia over a noncontextual structured template. A-D, Axial and coronal contrast-enhanced fat-suppressed imaging in a patient with skin cancer shows extensive perineural enhancement (arrows). E, Axial fat-suppressed T2-weighted image shows denervation atrophy in the right muscles of mastication (arrows). A noncontextual structured template $(F)$ and a contextual structured template $(G)$ describe the findings. Note that the noncontextual template is unnecessarily lengthy, and the skull base field is the only field conceivably relevant to trigeminal neuralgia, as opposed to the multiple relevant fields within the concise contextual template. Furthermore, discussion of the inferior alveolar nerve enhancement and muscular denervation atrophy could have been overlooked in the noncontextual template because there are no fields to describe them.

\section{Additional Contextual Templates}

In addition to the listed templates in this article, several other contextual templates were created (On-line Appendix). We made the following contextual adult brain MR imaging templates: rou- tine brain, stroke/TIA, headache, epilepsy, sella, orbits, cranial nerves III-IV-VI, cranial nerve VII, hearing loss, vestibular schwannoma follow-up, cholesteatoma, skull base, paranasal sinus, and temporomandibular joints. We created the following 

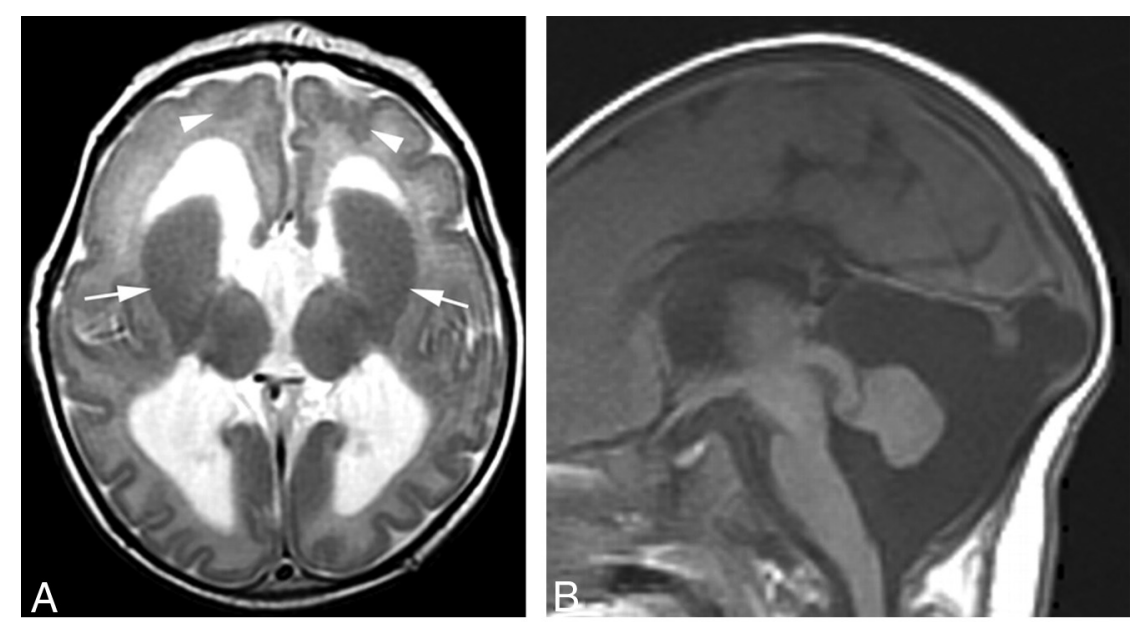

CLINICAL HISTORY: 6 wk old, term, developmental delay, microcephaly, seizures

FINDINGS:

BRAIN PARENCHYMA: No acute infarct or hemorrhage. Markedly enlarged caudate with absent internal capsule, resulting in fused striata bilaterally.

CORTEX: Bifrontal band heterotopias are seen as well as bioccipital pachygyria.

SULCATION: Delayed for age.

WHITE MATTER: Reduced volume. Myelination is delayed for age.

CORPUS CALLOSUM: Thinned.

VENTRICLES: Enlarged.

CEREBELLUM: Hypoplastic.

VERMIS: Hypoplastic.

BRAINSTEM: Hypoplastic pons.

MRS:

NAA:choline ratio: Normal.

Lactate Peak: Absent.

\section{IMPRESSION:}

Combination of abnormal basal ganglia, malformations of cortical development, microcephaly, and a small cerebellum/brainstem are compatible with a tublinopathy. C

FIG 6. Contextual template for developmental delay. A, Axial T2-weighted image of the brain shows fused corpus striata (arrows) and bifrontal band heterotopia (arrowheads). B, Sagittal T1-weighted image shows a hypoplastic corpus callosum, pons, and cerebellum. The contextual template $(C)$ arrives at the rare diagnosis of a tubulopathy by systematically inspecting each of the important structures in a patient with developmental delay and synthesizing them together. This method aids in diagnosis and can also teach trainees how to approach challenging cases.

contextual pediatric brain MR imaging templates: routine brain; fast protocol; epilepsy; and posterior fossa malformations, hemangiomas, arterial anomalies, cardiac defects, and eye abnormalities (PHACE syndrome). Contextual MR imaging neck templates included neck pain/swelling, cancer follow-up, and Horner syndrome. Contextual spine templates included the following: multiple sclerosis initial and follow-up, brachial and lumbosacral plexus, tethered cord, drop metastases, thoracic pain, and sagittal screening MR imaging. CT head contextual templates included the following: acute injury/ischemia, sinus, temporal bone, preoperative cochlear implant, CT angiogram head/neck, and CT venogram. 4D parathyroid adenoma and neck pain/swelling contextual templates were created for the CT neck category.

\section{Analysis of Contextual Templates}

To test the qualitative benefits of our contextual structured reports, we anonymously surveyed referring providers and radiologists in our hospital network (Tables 1 and 2). Referring providers consisted of neurologists, otolaryngologists, radiation oncologists, and neurosurgeons. Ninety-three percent of surveyed providers favored the contextual structured reports (either strongly agreed or agreed). Ninety-five percent of providers thought the pertinent findings were easy to understand, and $96 \%$ thought the impression was easy to understand. When asked how the reports compared with traditional narrative reporting, $83 \%$ preferred the contextual structured reports. In an optional commentary section of the survey, some providers highly praised our effort at standardization and uniformity, and other providers were pleased that certain important elements relevant to a specific disease process were always addressed, limiting second opinion communications.

For the survey of radiologists, both neuroradiologists and non-neuroradiologists were surveyed regarding contextual neuroradiology structured reports because non-neuroradiologists interpret a small percentage of neuroradiology examinations in our hospital network. Sixty-five percent of surveyed radiologists favored the contextual reports, while $24 \%$ were neutral and $11 \%$ were dissatisfied. Sixty percent thought the contextual templates made reporting more thorough and relevant for complex entities, $55 \%$ wanted contextual reports to be created for other radiology subspecialties, and $53 \%$ thought that the contextual reports made their reporting more efficient. When asked if he or she thought radiology report standardization was necessary, $66 \%$ of respondents agreed.

In our study, the referring providers' satisfaction was greater than radiologists' satisfaction, which has also been witnessed in other publications. ${ }^{8,31-33}$ One of these publications surveyed 265 academic radiologists from the United States, and only $60 \%$ were 
Table 1: Referring provider survey on contextual structured reporting $(n=85)^{a}$

\begin{tabular}{|c|c|c|c|c|c|}
\hline Question & $\begin{array}{l}\text { Strongly } \\
\text { Agree }\end{array}$ & Agree & $\begin{array}{l}\text { Neither Agree } \\
\text { nor Disagree }\end{array}$ & Disagree & $\begin{array}{l}\text { Strongly } \\
\text { Disagree }\end{array}$ \\
\hline $\begin{array}{l}\text { Do you have a positive overall impression of the new disease-specific } \\
\text { structured radiology reports as they pertain to your specialty? }\end{array}$ & $64(75)$ & $15(18)$ & $4(5)$ & $2(2)$ & $0(0)$ \\
\hline $\begin{array}{l}\text { On average, it is easy for me to understand the pertinent findings } \\
\text { from the structured radiology reports }\end{array}$ & $58(68)$ & $23(27)$ & $3(4)$ & $1(1)$ & $0(0)$ \\
\hline $\begin{array}{l}\text { On average, it is easy for me to understand the main impression from } \\
\text { the structured radiology reports }\end{array}$ & $55(65)$ & $26(31)$ & $3(4)$ & $1(1)$ & $0(0)$ \\
\hline $\begin{array}{l}\text { Do you prefer structured radiology reporting to the previous prose, } \\
\text { narrative reporting style you are accustomed to? }\end{array}$ & $52(61)$ & $19(22)$ & $13(15)$ & $1(1)$ & $0(0)$ \\
\hline
\end{tabular}

${ }^{a}$ Thirty-eight neurologists, 34 otolaryngologists, 10 radiation oncologists, and 3 neurosurgeons. Data are number (percentage).

Table 2: Radiologist survey on contextual structured reporting $(n=100)^{\mathrm{a}}$

\begin{tabular}{|c|c|c|c|c|c|}
\hline Question & $\begin{array}{l}\text { Strongly } \\
\text { Agree }\end{array}$ & Agree & $\begin{array}{c}\text { Neither Agree } \\
\text { nor Disagree }\end{array}$ & Disagree & $\begin{array}{l}\text { Strongly } \\
\text { Disagree }\end{array}$ \\
\hline $\begin{array}{l}\text { Do you have a positive overall impression of the new disease-specific } \\
\text { structured radiology reports as they pertain to your specialty? }\end{array}$ & $33(33)$ & $32(32)$ & $24(24)$ & $8(8)$ & $3(3)$ \\
\hline $\begin{array}{l}\text { Do you feel that using contextual structured neuroradiology } \\
\text { templates for complex entities makes your reporting more } \\
\text { thorough and relevant? }\end{array}$ & $28(28)$ & $32(32)$ & $29(29)$ & $7(7)$ & $4(4)$ \\
\hline $\begin{array}{l}\text { Do you think that the contextual structured reports increase your } \\
\text { efficiency? }\end{array}$ & $22(22)$ & $30(30)$ & $20(20)$ & $23(23)$ & $5(5)$ \\
\hline $\begin{array}{l}\text { Would you like contextual structured reports to be created for other } \\
\text { radiology subspecialties (eg, body, musculoskeletal, and so forth)? }\end{array}$ & $28(28)$ & $27(27)$ & $24(24)$ & $14(14)$ & $7(7)$ \\
\hline Do you think radiology report standardization is necessary? & $27(27)$ & $39(39)$ & $15(15)$ & $16(16)$ & $3(3)$ \\
\hline
\end{tabular}

satisfied with structured reports, whereas $27 \%$ were neutral and $13 \%$ were dissatisfied. ${ }^{32}$ We suspect that some of our radiologists were not in favor of the contextual reports because they were also not in favor of report standardization: Approximately one-third of respondents did not favor report standardization, and onethird did not favor the contextual reports. By filtering the data to those respondents who favored report standardization, the survey question percentages markedly improved. Eighty-eight percent of radiologists favored contextual reporting, 80\% thought the contextual templates made reporting more thorough and relevant for complex entities, $79 \%$ wanted contextual reports to be created for other radiology subspecialties, and $71 \%$ thought the contextual templates increased their efficiency. Thus, our data show that most radiologists who believe in report standardization also favored the contextual templates.

One potential reason that some radiologists did not believe in report standardization and contextual reporting is familiarity with narrative reporting. Narrative reporting has existed since the early 1900s and naturally has become ingrained in our profession. ${ }^{34}$ Other potential reasons for radiologists' dissatisfaction could be that structured reporting restricts autonomy, potentially undermines curiosity, and limits personalization. ${ }^{6,35}$ Despite these findings, we believe that radiologists' satisfaction will continue to increase as familiarity with contextual reports increases. Moreover, our stakeholders, the referring providers, demanding structured reporting will also be a great incentive.

Most radiologists who believed in report standardization also believed that contextual reporting increased their efficiency. This increased speed is likely because all the major items are discussed within the contextual template, thereby limiting actual dictation. We also observed a unique advantage of contextual reporting with normal or near-normal study findings, in that the template can be quickly signed. Our dictation software has a feature to default all the fields with normal findings using a simple voice command. The default values within each template field were established when each template was created. With regard to noncontextual structured templates, theoretically, these reports can also be signed quickly in the setting of normal examination findings, but the content is less meaningful.

Contextual reporting and our article have limitations. One potential limitation to contextual structured reporting is the phenomenon of "eye dwell," in which the radiologist is inclined to keep his or her eye on the report template rather than the images. ${ }^{6}$ We believe that this issue can occur in all structured reporting but should resolve with time after the radiologist becomes more accustomed to the template. Another limitation is when a patient has 2 disease entities, making it difficult to choose which contextual template to follow. In these cases, the single best template can be used and supplemented with additional content either in the "Other" section of the report or by inserting phrases from another template. With regard to the article, we did not measure radiologists' compliance in using the templates. While we encourage and advocate the use of the aforementioned templates, we are unable to force radiologists to use a specific template at our institution.

\section{Future Directions}

Because this proof of concept was favorable with the neuroradiology contextual templates, our group is planning to develop contextual templates for other radiology subspecialty diseases. Our group believes that contextual reporting is the next generation of structured reporting.

Contextual reporting could theoretically have a substantial role in data tracking and machine learning. Because contextual reports are disease-specific and structured, common data elements can be gathered from the reports so that computers can read and understand the content. These natural language-pro- 
cessing techniques could permit data extraction to populate national registries and aid research. ${ }^{36,37}$ Furthermore, contextual reports could also potentially be the framework for computergenerated reports if machine learning can reliably interpret certain radiologic examinations. Further studies are needed to substantiate these ideas.

\section{CONCLUSIONS}

Contextual reporting is a method of structured reporting specifically related to the disease or examination indication. Contextual templates are individually tailored to the diagnosis, they ensure that all pertinent points are addressed in a checklist fashion, and they educate trainees by providing a systematic approach for clinical interpretation. The included library of neuroradiology contextual templates can help neuroradiologists transition to a new method of structured reporting.

\section{REFERENCES}

1. Kahn CE Jr, Langlotz CP, Burnside ES, et al. Toward best practices in radiology reporting. Radiology 2009;252:852-56 CrossRef Medline

2. Radiological Society of North America Radiology Reporting Initiative. https://www.rsna.org/Reporting_Initiative.aspx. Accessed January 5, 2018

3. Radiological Society of North America Informatics Reporting. www.radreport.org. Accessed January 5, 2018

4. Bosmans JM, Peremans L, Menni M, et al. Structured reporting: if, why, when, how-and at what expense? Results of a focus group meeting of radiology professionals from eight countries. Insights Imaging 2012;3:295-302 CrossRef Medline

5. Douglas PS, Hendel RC, Cummings JE, et al; American College of Cardiology Foundation (ACCF). ACCF/ACR/AHA/ASE/ASNC/ HRS/NASCI/RSNA/SAIP/SCAI/SCCT/SCMR 2008 Health Policy Statement on Structured Reporting in Cardiovascular Imaging. J Am Coll Cardiol 2009;53:76-90 CrossRef Medline

6. Ganeshan D, Duong PT, Probyn L, et al. Structured reporting in radiology. Acad Radiol 2018;25:66-73 CrossRef Medline

7. Langlotz CP. Structured radiology reporting: are we there yet? $R a$ diology 2009;253:23-25 CrossRef Medline

8. Schwartz LH, Panicek DM, Berk AR, et al. Improving communication of diagnostic radiology findings through structured reporting. Radiology 2011;260:174-81 CrossRef Medline

9. Hawkins CM, Hall S, Hardin J, et al. Prepopulated radiology report templates: a prospective analysis of error rate and turnaround time. J Digit Imaging 2012;25:504-11 CrossRef Medline

10. Duszak R Jr, Nossal M, Schofield L, et al. Physician documentation deficiencies in abdominal ultrasound reports: frequency, characteristics, and financial impact. J Am Coll Radiol 2012;9:403-08 CrossRef Medline

11. Brook OR, Brook A, Vollmer CM, et al. Structured reporting of multiphasic CT for pancreatic cancer: potential effect on staging and surgical planning. Radiology 2015;274:464-72 CrossRef Medline

12. Radiological Society of North America Informatics Reporting. MR Brain Template. http://www.radreport.org/txt-mrrt/0000045. Accessed January 5, 2018

13. Boland GW, Duszak R Jr. Structured reporting and communication. J Am Coll Radiol 2015;12:1286-88 CrossRef Medline

14. Johnson AJ, Chen MY, Swan JS, et al. Cohort study of structured reporting compared with conventional dictation. Radiology 2009; 253:74-80 CrossRef Medline

15. Sabel BO, Plum JL, Kneidinger N, et al. Structured reporting of CT examinations in acute pulmonary embolism. J Cardiovasc Comput Tomogr 2017;11:188-95 CrossRef Medline

16. Wildman-Tobriner B, Allen BC, Davis JT, et al. Structured reporting of magnetic resonance enterography for pediatric Crohn's disease: effect on key feature reporting and subjective assessment of disease by referring physicians. Curr Probl Diagn Radiol 2017;46:110-14 CrossRef Medline

17. Magnetta MJ, Donovan AL, Jacobs BL, et al. Evidence-based reporting: a method to optimize prostate MRI communications with referring physicians. AJR Am J Roentgenol 2018;210:108-12 CrossRef Medline

18. KSAR Study Group for Rectal Cancer. Essential Items for Structured Reporting of Rectal Cancer MRI: 2016 Consensus Recommendation from the Korean Society of Abdominal Radiology. Korean J Radiol 2017;18:132-51 CrossRef Medline

19. Montoliu-Fornas G, Martí-Bonmatí L. Magnetic resonance imaging structured reporting in infertility. Fertil Steril 2016;105:1421-31 CrossRef Medline

20. Sverzellati N, Odone A, Silva M, et al; Italian Structured Report on Fibrosing Lung Disease Consort. Structured reporting for fibrosing lung disease: a model shared by radiologist and pulmonologist. $R a$ diol Med 2018;123:245-53 CrossRef Medline

21. Dickerson E, Davenport MS, Syed F, et al; Michigan Radiology Quality Collaborative. Effect of template reporting of brain MRIs for multiple sclerosis on report thoroughness and neurologist-rated quality: results of a prospective quality improvement project. $\mathrm{JAm}$ Coll Radiol 2017;14:371-79.e1 CrossRef Medline

22. Alessandrino F, Pichiecchio A, Mallucci G, et al. Do MRI structured reports for multiple sclerosis contain adequate information for clinical decision making? AJR Am J Roentgenol 2018;210:24-29 CrossRef Medline

23. American College of Radiology. Reporting and Data Systems. https:// www.acr.org/Clinical-Resources/Reporting-and-Data-Systems. Accessed January 5, 2018

24. Goldberg-Stein S, Walter WR, Amis ES, et al. Implementing a structured reporting initiative using a collaborative multistep approach. Curr Probl Diagn Radiol 2017;46:295-99 CrossRef Medline

25. Larson DB, Towbin AJ, Pryor RM, et al. Improving consistency in radiology reporting through the use of department-wide standardized structured reporting. Radiology 2013;267:240-50 CrossRef Medline

26. Rovira À, Wattjes MP, Tintoré M, et al; MAGNIMS study group. Evidence-based guidelines: MAGNIMS consensus guidelines on the use of MRI in multiple sclerosis-clinical implementation in the diagnostic process. Nat Rev Neurol 2015;11:471-82 CrossRef Medline

27. Filippi M, Rocca MA, Ciccarelli O, et al; MAGNIMS study group. MRI criteria for the diagnosis of multiple sclerosis: MAGNIMS consensus guidelines. Lancet Neurol 2016;15:292-303 CrossRef Medline

28. Deutschmann MW, Yeung J, Bosch M, et al. Radiologic reporting for paranasal sinus computed tomography: a multi-institutional review of content and consistency. Laryngoscope 2013;123:1100-05 CrossRef Medline

29. Sistrom C, Lanier L, Mancuso A. Reporting instruction for radiology residents. Acad Radiol 2004;11:76-84 CrossRef Medline

30. Lin E, Powell DK, Kagetsu NJ. Efficacy of a checklist-style structured radiology reporting template in reducing resident misses on cervical spine computed tomography examinations. J Digit Imaging 2014;27:588-93 CrossRef Medline

31. Weiss DL, Langlotz CP. Structured reporting: patient care enhancement or productivity nightmare? Radiology 2008;249:739-47 CrossRef Medline

32. Powell DK, Silberzweig JE. State of structured reporting in radiology: a survey. Acad Radiol 2015;22:226-33 CrossRef Medline

33. Faggioni L, Coppola F, Ferrari R, et al. Usage of structured reporting in radiological practice: results from an Italian online survey. Eur Radiol 2017;27:1934-43 CrossRef Medline

34. Reiner BI, Knight N, Siegel EL. Radiology reporting, past, present, and future: the radiologist's perspective. J Am Coll Radiol 2007;4: 313-19 CrossRef Medline

35. Gunderman $\mathrm{RB}, \mathrm{McNeive} \mathrm{LR}$. Is structured reporting the answer? Radiology 2014;273:7-9 CrossRef Medline

36. Rubin DL, Kahn CE Jr. Common data elements in radiology. Radiology 2017;283:837-44 CrossRef Medline

37. Chen MC, Ball RL, Yang L, et al. Deep learning to classify radiology free-text reports. Radiology 2018;286:845-52 CrossRef Medline 